PROCEEDINGS OF THE

AMERICAN MATHEMATICAL SOCIETY

Volume 132, Number 2, Pages 523-534

S 0002-9939(03)07051-5

Article electronically published on June 5, 2003

\title{
STRONG MIXING COEFFICIENTS FOR NON-COMMUTATIVE GAUSSIAN PROCESSES
}

\author{
WŁODZIMIERZ BRYC AND VICTOR KAFTAL
}

(Communicated by David R. Larson)

\begin{abstract}
Bounds for non-commutative versions of two classical strong mixing coefficients for $q$-Gaussian processes are found in terms of the angle between the underlying Hilbert spaces. As a consequence, we construct a $\psi$-mixing $q$ Gaussian stationary sequence with growth conditions on variances of partial sums. If classical processes with analogous properties were to exist, they would provide a counter-example to the Ibragimov conjecture.
\end{abstract}

\section{INTRODUCTION}

The long-standing Ibragimov conjecture in (classical) probability ([13], [12, and [9, Section 13.1]) involves the validity of the Central Limit Theorem for a stationary sequence of random variables $X_{k}$ which are $\phi$-mixing, i.e., such that there is a sequence $\phi_{N} \rightarrow 0$ such that for every $N, m, n \in \mathbb{N}$,

$$
\left|\operatorname{cov}\left(V_{1}, V_{2}\right)\right| \leq \phi_{N}\left\|V_{1}\right\|_{1}\left\|V_{2}\right\|_{\infty}
$$

for all bounded random variables $V_{1}, V_{2}$ such that $V_{1}$ is $\sigma\left(X_{1}, \ldots, X_{n}\right)$-measurable and $V_{2}$ is $\sigma\left(X_{n+N}, \ldots, X_{m+n+N}\right)$-measurable. Related to the Ibragimov conjecture are Bradley's conjecture [8] page 226], Iosifescu's conjecture [14], and works by M. Peligrad [18, and Berkes and Philipp [1].

Here we investigate the same notions in the non-commutative setting introduced by Voiculescu [19] for the free probability case $(q=0)$, and by Bozejko and Speicher [6] in the $-1<q<1$ case. Many classical (i.e., commutative) probability results have already been extended to these settings. In this paper we obtain a result, Theorem 4, which does not yet have a classical precursor. If a classical version of this theorem were to hold, it would settle in the negative Ibragimov's conjecture and all the other mentioned conjectures ([7]).

Non-commutative $q$-Gaussian random variables

$$
\mathbf{X}_{h}:=\mathbf{a}_{h}+\mathbf{a}_{h}^{*}
$$

are defined in terms of a bounded real-linear mapping $\mathbf{a}: \mathbb{H} \mapsto \mathcal{B}\left(\mathcal{H}_{q}\right)$ from a real Hilbert space $\mathbb{H}$ into the algebra of all bounded operators on a complex separable

Received by the editors September 12, 2002.

2000 Mathematics Subject Classification. Primary 81S05; Secondary 60E99.

Key words and phrases. Non-commutative uniform strong mixing, Ibragimov's conjecture, covariance estimates. 
Hilbert space $\mathcal{H}_{q}$ that satisfies the $q$-commutation relations

$$
\mathbf{a}_{g} \mathbf{a}_{h}^{*}-q \mathbf{a}_{h}^{*} \mathbf{a}_{g}=\langle h \mid g\rangle \mathbf{I}
$$

which were introduced in [11].

The von Neumann algebra $\mathcal{A}$ generated by these variables $\mathbf{X}_{h}$ (i.e., the weakoperator limits of non-commutative polynomials in the variables $\mathbf{X}_{h}$ ) has a tracial state $\mathbb{E}$. For $1 \leq p<\infty$, this trace permits us to define the $L_{p}$-norms

$$
\|\mathbf{X}\|_{p}:=\left(\mathbb{E}\left(\left(\mathbf{X}^{*} \mathbf{X}\right)^{p / 2}\right)\right)^{1 / p}
$$

and the non-commutative $L_{p}$ space $L_{p}(\mathcal{A}, \mathbb{E})$ is the closure of the von Neumann algebra $\mathcal{A}$ in this norm; see [17, Section 3]. We also use the standard conventions: $L_{\infty}(\mathcal{A}, \mathbb{E})$ is $\mathcal{A}$ with the operator norm and $L_{2}(\mathcal{A}, \mathbb{E})$ is a Hilbert space with the scalar product $(\mathbf{Y} \mid \mathbf{X}):=\mathbb{E}\left(\mathbf{X}^{*} \mathbf{Y}\right)$.

The main results we obtain are as follows. We first extend to the non-commutative setting a theorem of Kolmogorov and Rozanov [16] stating that for classical Gaussian sequences the "linear dependence coefficients" coincide with the "maximal correlation coefficients". In our setting, the linear dependence coefficient $r$ of two subspaces $\mathbb{H}_{1}, \mathbb{H}_{2} \subset \mathbb{H}$ is defined as

$$
r=r\left(\mathbb{H}_{1}, \mathbb{H}_{2}\right):=\sup \left\{\frac{\left|\operatorname{cov}\left(\mathbf{X}_{f}, \mathbf{X}_{g}\right)\right|}{\left\|\mathbf{X}_{f}\right\|_{2}\left\|\mathbf{X}_{g}\right\|_{2}}: \mathbf{X}_{f} \neq 0, \mathbf{X}_{g} \neq 0, f \in \mathbb{H}_{1}, g \in \mathbb{H}_{2}\right\} ;
$$

compare [9, Section 8.7]. Here,

$$
\operatorname{cov}(\mathbf{X}, \mathbf{Y}):=\mathbb{E}\left(\mathbf{X}^{*} \mathbf{Y}\right)-\mathbb{E}\left(\mathbf{X}^{*}\right) \mathbb{E}(\mathbf{Y}) .
$$

If $\mathcal{A}_{1}$ and $\mathcal{A}_{2}$ are the von Neumann algebras generated by $\left\{\mathbf{X}_{f}: f \in \mathbb{H}_{1}\right\}$ and $\left\{\mathbf{X}_{g}: g \in \mathbb{H}_{2}\right\}$ respectively, then the maximal correlation coefficient is

$$
\rho\left(\mathbb{H}_{1}, \mathbb{H}_{2}\right):=\sup \left\{\frac{|\operatorname{cov}(\mathbf{X}, \mathbf{Y})|}{\|\mathbf{X}\|_{2}\|\mathbf{Y}\|_{2}}: \mathbf{X} \neq 0, \mathbf{Y} \neq 0, \mathbf{X} \in L_{2}\left(\mathcal{A}_{1}, \mathbb{E}\right), \mathbf{Y} \in L_{2}\left(\mathcal{A}_{2}, \mathbb{E}\right)\right\} \text {. }
$$

\section{Theorem 1.}

$$
\rho\left(\mathbb{H}_{1}, \mathbb{H}_{2}\right)=r\left(\mathbb{H}_{1}, \mathbb{H}_{2}\right) .
$$

We then obtain an upper bound for the non-commutative analog of the $\psi$-mixing coefficient

$$
\psi\left(\mathbb{H}_{1}, \mathbb{H}_{2}\right):=\sup \left\{\frac{|\operatorname{cov}(\mathbf{X}, \mathbf{Y})|}{\|\mathbf{X}\|_{1}\|\mathbf{Y}\|_{1}}: \mathbf{X} \neq 0, \mathbf{Y} \neq 0, \mathbf{X} \in L_{2}\left(\mathcal{A}_{1}, \mathbb{E}\right), \mathbf{Y} \in L_{2}\left(\mathcal{A}_{2}, \mathbb{E}\right)\right\}
$$

(cf. 9, Theorem 3.10]). This result is somewhat unexpected since for the classical Gaussian random variables the $\psi$-mixing coefficient can only be zero (independent case) or infinity.

Theorem 2. If $r=r\left(\mathbb{H}_{1}, \mathbb{H}_{2}\right)<1$, then

$$
\psi\left(\mathbb{H}_{1}, \mathbb{H}_{2}\right) \leq C_{q}^{2} r \frac{r^{2}-3 r+4}{(1-r)^{3}},
$$

where $C_{q}=\prod_{m=1}^{\infty}\left(1-|q|^{m}\right)^{-3 / 2}$.

This upper bound is sharp in the free probability case, i.e., if $q=0$; for a related result, see also [2, Corollary 3].

Theorem 3. If $q=0$ and $r=r\left(\mathbb{H}_{1}, \mathbb{H}_{2}\right)<1$, then $\psi\left(\mathbb{H}_{1}, \mathbb{H}_{2}\right)=r \frac{r^{2}-3 r+4}{(1-r)^{3}}$. 
As a consequence of Theorem 2 we can adapt a classical probability construction of Bradley [7] to obtain the following non-commutative result.

Theorem 4. For every $\epsilon>0$ and $-1<q<1$ there exists a q-Gaussian sequence $\left\{\mathbf{X}_{k}\right\}$ such that the following statements hold true:

(i) $\mathbb{E}\left(\mathbf{X}_{j}\right)=0,\left\|\mathbf{X}_{1}+\cdots+\mathbf{X}_{n}\right\|_{2} \rightarrow \infty$ as $n \rightarrow \infty$, and $\frac{1}{n}\left\|\mathbf{X}_{1}+\cdots+\mathbf{X}_{n}\right\|_{2}^{2} \rightarrow 0$ as $n \rightarrow \infty$.

(ii) $\left\{\mathbf{X}_{k}\right\}$ is strictly stationary, i.e.,

$$
\mathbb{E}\left(\mathbf{X}_{i(1)} \ldots \mathbf{X}_{i(m)}\right)=\mathbb{E}\left(\mathbf{X}_{i(1)+t} \ldots \mathbf{X}_{i(m)+t}\right)
$$

for all $t, m \in \mathbb{N}$, and all sequences of integers $i(1), i(2), \ldots, i(m) \in \mathbb{N}$.

(iii) $\left\{\mathbf{X}_{k}\right\}$ is $\psi$-mixing, i.e., there is a monotone sequence of numbers $\psi_{N} \rightarrow 0$ such that $0<\psi_{1}<\epsilon$, and for all $m, n, N \in \mathbb{N}$,

$$
\left|\operatorname{cov}\left(\mathbf{V}_{1}, \mathbf{V}_{2}\right)\right| \leq \psi_{N}\left\|\mathbf{V}_{1}\right\|_{1}\left\|\mathbf{V}_{2}\right\|_{1}
$$

for all random variables $\mathbf{V}_{1}$ in the von Neumann algebra generated by $\mathbf{X}_{1}, \ldots, \mathbf{X}_{n}$, and $\mathbf{V}_{2}$ in the von Neumann algebra generated by $\mathbf{X}_{n+N}, \ldots, \mathbf{X}_{m+n+N}$.

Our proof of Theorem 2 is based on the proof of Theorem 1 and, via a duality argument, on the main theorem in Bozejko [4]. In the free case which corresponds to $q=0$, a more self-contained proof along the lines of [3] is given in Section [3] where we also present the proof of Theorem 3 .

\section{Proofs}

We will be working with the $q$-Fock space representation of $q$-Gaussian processes, adapted from [5]; see also [19 Section 1.5] for the $q=0$ (free) case. For a real Hilbert space $\mathbb{H}$ with complexification $\mathbb{H}_{c}:=\mathbb{H} \oplus i \mathbb{H}$, the associated $q$-Fock space $\mathcal{H}_{q}$ is the closure of $\bigoplus_{n=0}^{\infty} \mathbb{H}_{c}^{\otimes n}$ with respect to the scalar product obtained as the sesquilinear extension of

$$
\left\langle g_{1} \otimes \cdots \otimes g_{n} \mid h_{1} \otimes \cdots \otimes h_{m}\right\rangle_{q}= \begin{cases}\sum_{\sigma \in S_{n}} q^{|\sigma|} \prod_{j=1}^{n}\left\langle g_{j} \mid h_{\sigma(j)}\right\rangle & \text { if } m=n \\ 0 & \text { if } m \neq n .\end{cases}
$$

Here, $\mathbb{H}_{c}^{\otimes 0}:=\mathbb{C} \mathbf{1}$, where $\mathbf{1}$ is called the vacuum vector, $S_{n}$ is the set of all the permutations of $\{1, \ldots, n\}$ and $|\sigma|:=\operatorname{card}\{(i, j): i<j, \sigma(i)>\sigma(j)\}$ is the number of inversions of $\sigma \in S_{n}$.

We denote by $\|\cdot\|_{\mathcal{H}_{q}}$ the corresponding norm. We denote by $\overline{\mathbb{H}} \otimes n$ the $\|\cdot\|_{\mathcal{H}_{q}}{ }^{-}$ closure of the algebraic tensor product $\mathbb{H}_{c}^{\otimes n}$ so that $\mathcal{H}_{q}=\bigoplus_{n=0}^{\infty} \overline{\mathbb{H} \otimes n}$. In this setting, for $h \in \mathbb{H}$, the annihilation operator $\mathbf{a}_{h}: \mathcal{H}_{q} \rightarrow \mathcal{H}_{q}$ and its adjoint, the creation operator $\mathbf{a}_{h}^{*}: \mathcal{H}_{q} \rightarrow \mathcal{H}_{q}$, are the bounded linear extensions of

$$
\begin{gathered}
\mathbf{a}_{h} \mathbf{1}:=0 \\
\mathbf{a}_{h} g_{1} \otimes \cdots \otimes g_{n}:=\sum_{j=1}^{n} q^{j-1}\left\langle h \mid g_{j}\right\rangle g_{1} \otimes \cdots \otimes g_{j-1} \otimes g_{j+1} \otimes \cdots \otimes g_{n}
\end{gathered}
$$

and

$$
\begin{gathered}
\mathbf{a}_{h}^{*} \mathbf{1}=h, \\
\mathbf{a}_{h}^{*} g_{1} \otimes \cdots \otimes g_{n}:=h \otimes g_{1} \otimes \cdots \otimes g_{n}
\end{gathered}
$$


for $g_{1}, g_{2}, \ldots, g_{n} \in \mathbb{H}_{c}$, and satisfy relations (2) (see [6], [5]; cf. also [19], Example 1.5.8] for $q=0)$.

Let $\mathcal{A}$ be the von Neumann algebra generated by the variables $\left\{\mathbf{X}_{h}: h \in \mathbb{H}\right\}$ given by (1). It is known that the vacuum expectation state $\mathbb{E}: \mathcal{A} \rightarrow \mathbb{C}$ defined by

$$
\mathbb{E}(\mathbf{X}):=\langle\mathbf{X} \mathbf{1} \mid \mathbf{1}\rangle_{\mathcal{H}_{q}}
$$

is a faithful normal finite trace on $\mathcal{A}$; see [5, Proposition 2.3], or [19, Theorem 2.6.2 (ii)] when $q=0$.

For $g_{1}, g_{2}, \ldots, g_{n} \in \mathbb{H}$, the Wick product $\Psi\left(g_{1} \otimes \cdots \otimes g_{n}\right) \in \mathcal{A}$ is defined recursively by $\Psi(\mathbf{1}):=\mathbf{I}, \Psi(h):=\mathbf{X}_{h}$, and

$$
\begin{gathered}
\Psi\left(h \otimes g_{1} \otimes \cdots \otimes g_{n}\right):=\mathbf{X}_{h} \Psi\left(g_{1} \otimes \cdots \otimes g_{n}\right) \\
-\sum_{j=1}^{n} q^{j-1}\left\langle h \mid g_{j}\right\rangle \Psi\left(g_{1} \otimes \cdots \otimes g_{j-1} \otimes g_{j+1} \otimes \cdots \otimes g_{n}\right) .
\end{gathered}
$$

By definition, $\mathbf{X}_{h} \mathbf{1}=h$, so

$$
\mathbb{E}\left(\mathbf{X}_{h}\right)=0
$$

and

$$
\left\|\mathbf{X}_{h}\right\|_{2}=\|h\|
$$

for all $h \in \mathbb{H}$.

By (11), $\Psi\left(h_{1} \otimes \cdots \otimes h_{n}\right)=\mathbf{X}_{h_{1}} \mathbf{X}_{h_{2}} \ldots \mathbf{X}_{h_{n}}+\cdots$, where the dots represent a polynomial in $\mathbf{X}_{h_{1}}, \ldots, \mathbf{X}_{h_{n}}$ of degree lower than $n$. Thus it is clear that every non-commutative polynomial in the variables $\mathbf{X}_{h_{1}}, \ldots, \mathbf{X}_{h_{n}}$ can be expressed as a linear combination of Wick products. We will need to make this relation more precise in Lemma 1

Denote by $\mathbf{i}$ the multi-index $\mathbf{i}:=(i(1), \ldots, i(N)) \in \mathbb{N}^{N}$ and denote by $|\mathbf{i}|$ the length $N$ of the multi-index $\mathbf{i}$. Let $(\mathbf{i}, \mathbf{j})$ denote the concatenation of the multiindices $\mathbf{i}, \mathbf{j}$ :

$$
(\mathbf{i}, \mathbf{j})=(i(1), i(2), \ldots, i(L), j(1),(j(2), \ldots, j(M))) .
$$

Thus $|(\mathbf{i}, \mathbf{j})|=|\mathbf{i}|+|\mathbf{j}|$. Denote by $\mathbf{i}[a \ldots b]$ the subindex $(i(a), i(a+1), \ldots, i(b))$.

For a sequence of vectors $g_{1}, g_{2}, \cdots \in \mathbb{H}$ write

$$
g^{\otimes \mathbf{i}}=g_{i(1)} \otimes g_{i(2)} \otimes \cdots \otimes g_{i(m)}
$$

so that $g^{\otimes(\mathbf{i}, \mathbf{j})}=g^{\otimes \mathbf{i}} \otimes g^{\otimes \mathbf{j}}$.

Lemma 1. For every $m \in \mathbb{N}$ and all multi-indices $\mathbf{i}$ of length $0 \leq|\mathbf{i}| \leq m$ there are polynomials $P_{\mathbf{i}}^{m}$ in $m^{2}$ variables $\left\{x_{i, j}: i, j \leq m\right\}$ such that for any $g_{1}, g_{2}, \ldots, g_{m} \in$ $\mathbb{H}$,

$$
\mathbf{X}_{g_{m}} \mathbf{X}_{g_{m-1}} \ldots \mathbf{X}_{g_{1}}=\sum_{|\mathbf{i}| \leq m} P_{\mathbf{i}}^{m}\left(x_{s, t}: s, t \leq m\right) \Psi\left(g^{\otimes \mathbf{i}}\right),
$$

where $x_{s, t}=\left\langle g_{s} \mid g_{t}\right\rangle$, and if $|\mathbf{i}|=0$, then $g^{\otimes \emptyset}=\mathbf{1}$.

Proof. We proceed by induction with respect to $m \geq 1$. If $m=1$, then $\mathbf{X}_{g_{1}}=\Psi\left(g_{1}\right)$ proving (14) with $P_{\emptyset}^{1}=0, P_{1}^{1}=1, P_{i}^{1}=0$ for $i>1$. 
Suppose that formula (14) holds true for some $m \in \mathbb{N}$. Then from (11) we get

$$
\begin{gathered}
\mathbf{X}_{g_{m+1}} \mathbf{X}_{g_{m}} \ldots \mathbf{X}_{g_{1}}=\sum_{|\mathbf{i}| \leq m} P_{\mathbf{i}}^{m}\left(x_{s, t}: s, t \leq m\right) \mathbf{X}_{g_{m+1}} \Psi\left(g^{\otimes \mathbf{i}}\right) \\
=\sum_{|\mathbf{i}| \leq m} P_{\mathbf{i}}^{m}\left(x_{s, t}: s, t \leq m\right) \Psi\left(g^{\otimes(m+1, \mathbf{i})}\right) \\
\left.+\sum_{|\mathbf{i}| \leq m} P_{\mathbf{i}}^{m}\left(x_{s, t}: s, t \leq m\right) \sum_{k=1}^{|\mathbf{i}|} q^{k-1}\left\langle g_{m+1}\right| g_{i(k)}\right) \Psi\left(g^{\otimes(\mathbf{i}[0 \ldots k-1], \mathbf{i}[k+1 \ldots|\mathbf{i}|])}\right) .
\end{gathered}
$$

Notice that in the last sum the same multi-index can be obtained from more than one concatenation $(\mathbf{i}[0 \ldots k-1], \mathbf{i}[k+1 \ldots|\mathbf{i}|])$. Grouping all of them together and noticing that $\left\langle g_{m+1} \mid g_{s}\right\rangle=x_{m+1, s}$, we get the polynomials in the right-hand side of (14).

From (11) and (2),

$$
\Psi\left(h_{1} \otimes \cdots \otimes h_{n}\right) \mathbf{1}=h_{1} \otimes \cdots \otimes h_{n},
$$

and thus $\left\|\Psi\left(h_{1} \otimes \cdots \otimes h_{n}\right)\right\|_{2}=\left\|h_{1} \otimes \cdots \otimes h_{n}\right\|_{\mathcal{H}_{q}}$, which extends (13). Therefore, the mapping

$$
\sum \alpha_{i_{1}, \ldots, i_{k}} h_{i_{1}} \otimes \cdots \otimes h_{i_{k}} \mapsto \sum \alpha_{i_{1}, \ldots, i_{k}} \Psi\left(h_{i_{1}} \otimes \cdots \otimes h_{i_{k}}\right)
$$

is an isometry in the $L_{2}$-norm (3) from a dense subset of $\mathcal{H}_{q}$ onto all the polynomials in $\left\{\mathbf{X}_{h}: h \in \mathbb{H}\right\}$ and hence it extends to a unitary mapping $\widetilde{\Psi}$ of $\mathcal{H}_{q}$ onto the Hilbert space $L_{2}(\mathcal{A}, \mathbb{E})$. Thus $\widetilde{\Psi}$ induces the orthogonal decomposition

$$
L_{2}(\mathcal{A}, \mathbb{E})=\bigoplus_{n=0}^{\infty} \widetilde{\Psi}(\overline{\mathbb{H} \otimes n})
$$

Furthermore,

$$
\widetilde{\Psi}(\xi) \mathbf{1}=\xi
$$

for all $\xi \in \mathcal{H}_{q}$.

Proof of Theorem 1 . First, we give a Hilbert space theoretic characterization of the linear dependence coefficient $r=r\left(\mathbb{H}_{1}, \mathbb{H}_{2}\right)$ refined by (4). By (12)

$$
\operatorname{cov}\left(\mathbf{X}_{f}, \mathbf{X}_{g}\right)=\mathbb{E}\left(\mathbf{X}_{f}^{*} \mathbf{X}_{g}\right)=\left(\mathbf{X}_{g} \mid \mathbf{X}_{f}\right)=(\widetilde{\Psi}(g) \mid \widetilde{\Psi}(f))=\langle f \mid g\rangle_{\mathcal{H}_{q}}=\langle f \mid g\rangle .
$$

Hence taking into account (13) we obtain

$$
r=\sup \left\{\langle f \mid g\rangle: f \in \mathbb{H}_{1}, g \in \mathbb{H}_{2},\|f\|_{\mathbb{H}}=\|g\|_{\mathbb{H}}=1\right\} .
$$

Now let $P_{j}: \mathbb{H}_{c} \rightarrow \mathbb{H}_{c}$ denote the orthogonal projection onto $\mathbb{H}_{j} \subset \mathbb{H}_{c}, \mathrm{j}=1,2$. It is easy to verify that $\left\|P_{1} P_{2}\right\|=r$.

The $n$-fold tensor product $P_{j}^{\otimes n}$ of the projection $P_{j}$ with itself is clearly a linear idempotent operator on $\mathbb{H}_{c}^{\otimes n}$. It is also selfadjoint with respect to the scalar 
product (8). Indeed, if $g_{1} \otimes g_{2} \otimes \cdots \otimes g_{n}$ and $h_{1} \otimes h_{2} \otimes \cdots \otimes h_{n}$ are in $\mathbb{H}_{c}^{\otimes n}$, then

$$
\begin{gathered}
\left\langle P_{j}^{\otimes n} g_{1} \otimes \cdots \otimes g_{n} \mid h_{1} \otimes \cdots \otimes h_{m}\right\rangle_{q}=\left\langle P_{j} g_{1} \otimes \cdots \otimes P_{j} g_{n} \mid h_{1} \otimes \cdots \otimes h_{m}\right\rangle_{q} \\
=\sum_{\sigma \in S_{n}} q^{|\sigma|} \prod_{k=1}^{n}\left\langle P_{j} g_{k} \mid h_{\sigma(k)}\right\rangle=\sum_{\sigma \in S_{n}} q^{|\sigma|} \prod_{k=1}^{n}\left\langle g_{k} \mid P_{j} h_{\sigma(k)}\right\rangle \\
=\left\langle g_{1} \otimes \cdots \otimes g_{n} \mid P_{j}^{\otimes n} h_{1} \otimes \cdots \otimes h_{m}\right\rangle_{q} .
\end{gathered}
$$

Moreover, it is easy to see that $P_{j}^{\otimes n}$, and hence $\left(P_{2} P_{1}\right)^{\otimes n}=P_{2}^{\otimes n} P_{1}^{\otimes n}$, commute with the unitary operations of permuting the components of $\mathbb{H}_{c}^{\otimes n}$. Therefore, by [5, Lemma 1.4], the norm $\left\|\left(P_{2} P_{1}\right)^{\otimes n}\right\|$ of $\left(P_{2} P_{1}\right)^{\otimes n}$ with respect to the norm $\|\cdot\|_{\mathcal{H}_{q}}$ coincides with the norm with respect to the Hilbert space tensor norm. Therefore, by [15. Section 2.6.12 Eqn. (16)] $\left\|\left(P_{2} P_{1}\right)^{\otimes n}\right\|=\left\|P_{1} P_{2}\right\|^{n}$, where $\left\|P_{1} P_{2}\right\|$ is the usual operator norm in $B\left(\mathbb{H}_{c}\right)$ which, as we observed above, coincides with $r$.

Thus for $n \geq 1, \xi \in \overline{\mathbb{H}_{1}^{\otimes n}}, \eta \in \overline{\mathbb{H}_{2}^{\otimes n}}$, we have

$$
\left|\mathbb{E}\left(\widetilde{\Psi}(\eta)^{*} \widetilde{\Psi}(\xi)\right)\right| \leq r^{n}\|\widetilde{\Psi}(\xi)\|_{2}\|\widetilde{\Psi}(\eta)\|_{2} .
$$

Indeed,

$$
\begin{aligned}
& \left|\mathbb{E}\left(\widetilde{\Psi}(\eta)^{*} \widetilde{\Psi}(\xi)\right)\right|=\left|\langle\widetilde{\Psi}(\xi) \mathbf{1} \mid \widetilde{\Psi}(\eta) \mathbf{1}\rangle_{\mathcal{H}_{q}}\right|=\left|\langle\xi \mid \eta\rangle_{\mathcal{H}_{q}}\right| \\
& \quad=\left|\left\langle P_{1}^{\otimes n} \xi \mid P_{2}^{\otimes n} \eta\right\rangle_{\mathcal{H}_{q}}\right|=\left|\left\langle P_{2}^{\otimes n} P_{1}^{\otimes n} \xi \mid \eta\right\rangle_{\mathcal{H}_{q}}\right|=\left|\left\langle\left(P_{2} P_{1}\right)^{\otimes n} \xi \mid \eta\right\rangle_{\mathcal{H}_{q}}\right| \\
& \quad \leq\left\|\left(P_{2} P_{1}\right)^{\otimes n}\right\|\|\xi\|_{\mathcal{H}_{q}}\|\eta\|_{\mathcal{H}_{q}}=r^{n}\|\xi\|_{\mathcal{H}_{q}}\|\eta\|_{\mathcal{H}_{q}}=r^{n}\|\widetilde{\Psi}(\xi)\|_{2}\|\widetilde{\Psi}(\eta)\|_{2},
\end{aligned}
$$

where the last equality follows because $\widetilde{\Psi}$ is an isometry.

Now denote by $\mathbf{X}^{(n)}, \mathbf{Y}^{(n)}$ the components of $\mathbf{X}, \mathbf{Y}$ in the direct sum decomposition (15). Since $\mathbf{X} \in L_{2}\left(\mathcal{A}_{1}, \mathbb{E}\right)$, then $\mathbf{X}^{(n)}$ is in the closed subspace $\widetilde{\Psi}\left(\overline{\mathbb{H}_{1}^{\otimes n}}\right)$ of $\widetilde{\Psi}\left(\overline{\mathbb{H}^{\otimes n}}\right)$, and similarly $\mathbf{Y}^{(n)} \in \widetilde{\Psi}\left(\overline{\mathbb{H}_{2}^{\otimes n}}\right)$ for all $n$. So from (18) we get for $n \geq 1$ that

$$
\left|\mathbb{E}\left(\mathbf{X}^{(n) *} \mathbf{Y}^{(n)}\right)\right| \leq r^{n}\left\|\mathbf{X}^{(n)}\right\|_{2}\left\|\mathbf{Y}^{(n)}\right\|_{2} .
$$

From (16) we see that $\mathbf{X}^{(n)} \mathbf{1} \in \overline{\mathbb{H}_{1}^{\otimes n}}$ and hence $\mathbb{E}\left(\mathbf{X}^{(n)}\right)=0$ for $n \geq 1$. It is easy to verify that $\mathbb{E}(\mathbf{X})=\mathbb{E}\left(\mathbf{X}^{(0)}\right), \mathbb{E}(\mathbf{Y})=\mathbb{E}\left(\mathbf{Y}^{(0)}\right)$, and $\mathbb{E}\left(\mathbf{X}^{(0)^{*}} \mathbf{Y}^{(0)}\right)=$ $\mathbb{E}\left(\mathbf{X}^{(0)^{*}}\right) \mathbb{E}\left(\mathbf{Y}^{(0)}\right)=\mathbb{E}(\mathbf{X}) \mathbb{E}(\mathbf{Y})$. Keeping in mind that $\mathbb{E}\left(\mathbf{X}^{*} \mathbf{Y}\right)$ is the scalar product of $\mathbf{Y}$ and $\mathbf{X}$ in $L_{2}(\mathcal{A}, \mathbb{E})$ we have

$$
\mathbb{E}\left(\mathbf{X}^{*} \mathbf{Y}\right)=\sum_{n=0}^{\infty} \mathbb{E}\left(\mathbf{X}^{(n) *} \mathbf{Y}^{(n)}\right)
$$

Therefore

$$
|\operatorname{cov}(\mathbf{X}, \mathbf{Y})|=\left|\mathbb{E}\left(\mathbf{X}^{*} \mathbf{Y}\right)-\mathbb{E}\left(\mathbf{X}^{*}\right) \mathbb{E}(\mathbf{Y})\right| \leq \sum_{n=1}^{\infty}\left|\mathbb{E}\left(\mathbf{X}^{(n) *} \mathbf{Y}^{(n)}\right)\right|,
$$

and inequality (19) gives

$$
|\operatorname{cov}(\mathbf{X}, \mathbf{Y})| \leq \sum_{n=1}^{\infty} r^{n}\left\|\mathbf{X}^{(n)}\right\|_{2}\left\|\mathbf{Y}^{(n)}\right\|_{2}
$$


As $r^{n} \leq r$, by the Cauchy-Schwarz inequality we have

$$
|\operatorname{cov}(\mathbf{X}, \mathbf{Y})| \leq r\left(\sum_{n=1}^{\infty}\left\|\mathbf{X}^{(n)}\right\|_{2}^{2}\right)^{1 / 2}\left(\sum_{n=1}^{\infty}\left\|\mathbf{Y}^{(n)}\right\|_{2}^{2}\right)^{1 / 2} \leq r\|\mathbf{X}\|_{2}\|\mathbf{Y}\|_{2},
$$

which proves the theorem.

Proof of Theorem $\mathbf{2}$. Let $\mathbf{X} \in L_{2}(\mathcal{A}, \mathbb{E})$. As in the proof of Theorem 1 , denote by $\mathbf{X}^{(n)}$ the $n$-th term in the expansion (15) of $\mathbf{X}$. Since $L_{2}(\mathcal{A}, \mathbb{E})$ is a Hilbert space,

$$
\left\|\mathbf{X}^{(n)}\right\|_{2}=\sup \left\{\left|\mathbb{E}\left(\mathbf{Z}^{*} \mathbf{X}^{(n)}\right)\right|: \mathbf{Z} \in L_{2}(\mathcal{A}, \mathbb{E}),\|\mathbf{Z}\|_{2} \leq 1\right\} .
$$

By (20), $\mathbb{E}\left(\mathbf{Z}^{*} \mathbf{X}^{(n)}\right)=\mathbb{E}\left(\mathbf{Z}^{(n) *} \mathbf{X}^{(n)}\right)=\mathbb{E}\left(\mathbf{Z}^{(n) *} \mathbf{X}\right)$, where $\mathbf{Z}^{(n)}$ is the component of $\mathbf{Z}$ in $\widetilde{\Psi}\left(\overline{\mathbb{H}^{\otimes n}}\right)$. As $\mathcal{A}$ is dense in $L_{2}(\mathcal{A}, \mathbb{E})$, we get

$$
\left\|\mathbf{X}^{(n)}\right\|_{2}=\sup \left\{\left|\mathbb{E}\left(\mathbf{Z}^{*} \mathbf{X}\right)\right|: \mathbf{Z} \in \mathcal{A} \cap \widetilde{\Psi}(\overline{\mathbb{H} \otimes n}),\|\mathbf{Z}\|_{2} \leq 1\right\} .
$$

For $\mathbf{Z} \in \mathcal{A} \cap \widetilde{\Psi}(\overline{\mathbb{H} \otimes n})$ and $\|\mathbf{Z}\|_{2} \leq 1$, by Hölder's inequality ([17] (23)]) we get

$$
\left|\mathbb{E}\left(\mathbf{Z}^{*} \mathbf{X}\right)\right| \leq\left\|\mathbf{Z}^{*}\right\|_{\infty}\|\mathbf{X}\|_{1}=\|\mathbf{Z}\|_{\infty}\|\mathbf{X}\|_{1} .
$$

By [4, Proposition 2.1(b)],

$$
\|\mathbf{Z}\|_{\infty} \leq C_{q}(n+1)\|\mathbf{Z}\|_{2} \leq C_{q}(n+1) .
$$

Hence

$$
\left\|\mathbf{X}^{(n)}\right\|_{2} \leq C_{q}(n+1)\|\mathbf{X}\|_{1} .
$$

The same inequality holds for any $\mathbf{Y} \in L_{2}(\mathcal{A}, \mathbb{E})$.

Applying these inequalities to each term on the right-hand side of (21) we get

$$
|\operatorname{cov}(\mathbf{X}, \mathbf{Y})| \leq C_{q}^{2} \sum_{n=1}^{\infty}(n+1)^{2} r^{n}\|\mathbf{X}\|_{1}\|\mathbf{Y}\|_{1}=C_{q}^{2} r \frac{r^{2}-3 r+4}{(1-r)^{3}}\|\mathbf{X}\|_{1}\|\mathbf{Y}\|_{1},
$$

which completes the proof.

Proof of Theorem 4 To prove this theorem, we need to construct an appropriate sequence of vectors $h_{k}$ in a real Hilbert space $\mathbb{H}$. The construction relies on [7] (and hence, indirectly, on results of Helson and Sarason on Toeplitz forms); according to [7 Lemma 3], for every $\epsilon>0$ there is a sequence $h_{k}$ of (real) classical Gaussian random variables on a probability space $(\Omega, \mathcal{F}, P)$ with the following properties:

(i') $\left\|h_{1}+\cdots+h_{n}\right\|_{2} \rightarrow \infty$ and $\frac{1}{n}\left\|h_{1}+\cdots+h_{n}\right\|_{2}^{2} \rightarrow 0$.

(ii') $\left\langle h_{t} \mid h_{t+m}\right\rangle=\left\langle h_{0} \mid h_{m}\right\rangle$ for all $m, t \in \mathbb{N}$.

(iii') There exists a monotone sequence $\epsilon_{N} \rightarrow 0$ such that $\epsilon_{1}<\min (1, \epsilon)$ and for every (real) linear combination $v_{1}=\sum_{j=1}^{n} a_{j} h_{j}, v_{2}=\sum_{j=n+N}^{n+N+m} b_{j} h_{j}$ we have

$$
\left|\left\langle v_{1} \mid v_{2}\right\rangle\right| \leq \epsilon_{N}\left\|v_{1}\right\|_{2}\left\|v_{2}\right\|_{2}
$$

where $\langle g \mid h\rangle$ is the scalar product in $L_{2}(\Omega, \mathcal{F}, P)$.

We define $\mathbb{H}$ as the closure of the real span of $h_{k}$ in $L_{2}(\Omega, \mathcal{F}, P)$. For any $-1<$ $q<1$, let $\mathcal{H}_{q}$ be the $q$-Fock space based on $\mathbb{H}$, with the creation and annihilation operators $\mathbf{a}_{h}, \mathbf{a}_{h}^{*}$ defined by (9), (10) and the $q$-Gaussian random variables $\mathbf{X}_{h}$ defined in (11). We now verify that the $q$-Gaussian sequence $\mathbf{X}_{k}:=\mathbf{X}_{h_{k}}$ has the properties (i)-(iii). 
Statement (i) follows from (i') by (12), and

$$
\left\|\mathbf{X}_{1}+\cdots+\mathbf{X}_{n}\right\|_{2}^{2}=\mathbb{E}\left(\left|\mathbf{X}_{1}+\cdots+\mathbf{X}_{n}\right|^{2}\right)=\mathbb{E}\left(\left|\mathbf{X}_{h_{1}+\cdots+h_{n}}\right|^{2}\right)=\left\|h_{1}+\cdots+h_{n}\right\|_{\mathbb{H}}^{2},
$$

where the second equality follows from the linearity of $\mathbf{a}: \mathbb{H} \mapsto \mathcal{B}\left(\mathcal{H}_{q}\right)$ ) and the third one holds true by (13).

Statement (ii) follows from (ii') as follows. Since $\mathbb{E}\left(\Psi\left(h^{\otimes \mathbf{i}}\right)\right)=0$ for $|\mathbf{i}|>0$, by (14)

$$
\mathbb{E}\left(\mathbf{X}_{i(1)+t} \ldots \mathbf{X}_{i(m)+t}\right)=P_{\emptyset}^{m}\left(x_{r, s}: r, s \leq m\right)
$$

is a polynomial in the $m^{2}$ variables $x_{r, s}=\left\langle h_{i(r)+t} \mid h_{i(s)+t}\right\rangle$. Since (ii') implies that $\left\langle h_{i(r)+t} \mid h_{i(s)+t}\right\rangle=\left\langle h_{i(r)} \mid h_{i(s)}\right\rangle, r, s \in \mathbb{N}$, therefore (7) follows.

Statement (iii) is a consequence of Theorem 2 and (iii'). In this context, fix $n, m, N \in \mathbb{N}$ and let $\mathbb{H}_{1}$ be spanned by vectors $\left\{h_{1}, \ldots, h_{n}\right\}$ and $\mathbb{H}_{2}$ be spanned by vectors $\left\{h_{n+N}, \ldots, h_{m+n+N}\right\}$. Thus by (17), we have $r\left(\mathbb{H}_{1}, \mathbb{H}_{2}\right) \leq \epsilon_{N}$. By (6) and the monotonicity in $r$ of the right-hand side of (6) we get (iii) with $\psi_{N}=$ $C_{q}^{2} \frac{4 \epsilon_{N}}{\left(1-\epsilon_{N}\right)^{3}}$.

\section{FREE PROCESSES}

Proof of Theorem 3. By Theorem $2, \psi\left(\mathbb{H}_{1}, \mathbb{H}_{2}\right) \leq r \frac{r^{2}-3 r+4}{(1-r)^{3}}$. Since $\psi\left(\mathbb{H}_{1}, \mathbb{H}_{2}\right) \geq 0$, we can assume without loss of generality that $0<r<1$. Fix $\epsilon \in(0, r)$. Then there are unit vectors $f \in \mathbb{H}_{1}, g \in \mathbb{H}_{2}$ such that $r_{0}:=\langle f \mid g\rangle>r-\epsilon>0$. Then

$$
\psi\left(\mathbb{H}_{1}, \mathbb{H}_{2}\right) \geq \sup \frac{\operatorname{cov}\left(v\left(\mathbf{X}_{f}\right), w\left(\mathbf{X}_{g}\right)\right)}{\left\|v\left(\mathbf{X}_{f}\right)\right\|_{1}\left\|w\left(\mathbf{X}_{g}\right)\right\|_{1}},
$$

where the supremum is taken over all real continuous functions $v, w$. The joint distribution of $\mathbf{X}_{f}, \mathbf{X}_{g}$ is known, and has the density

$$
p(x, y)=\frac{1-r_{0}^{2}}{4 \pi^{2}} \frac{\sqrt{4-x^{2}} \sqrt{4-y^{2}}}{\left(1-r_{0}^{2}\right)^{2}-r_{0}\left(1+r_{0}^{2}\right) x y+r_{0}^{2}\left(x^{2}+y^{2}\right)},
$$

i.e., $\mathbb{E}\left(v\left(\mathbf{X}_{f}\right) w\left(\mathbf{X}_{g}\right)\right)=\int_{-2}^{2} \int_{-2}^{2} v(x) w(y) p(x, y) d x d y$; see [5, Theorem 1.10]. The one-dimensional distributions of $\mathbf{X}_{f}, \mathbf{X}_{g}$ have the same density $p(x)=\frac{1}{2 \pi} \sqrt{4-x^{2}}$. Thus the right-hand side of (23) becomes

$$
\sup \frac{\left|\int v(x) w(y)(p(x, y)-p(x) p(y)) d x d y\right|}{\int|v(x)| p(x) d x \int|w(y)| p(y) d y}
$$

which is equal to

$$
\begin{aligned}
& \sup _{|x|,|y| \leq 2}\left|1-\frac{p(x, y)}{p(x) p(y)}\right| \\
& \quad=\sup _{|x|,|y| \leq 2}\left|1-\frac{1-r_{0}^{2}}{\left(1-r_{0}^{2}\right)^{2}-r_{0}\left(1+r_{0}^{2}\right) x y+r_{0}^{2}\left(x^{2}+y^{2}\right)}\right|=r_{0} \frac{r_{0}^{2}-3 r_{0}+4}{\left(1-r_{0}\right)^{3}} .
\end{aligned}
$$

Since $r-\epsilon<r_{0} \leq r$ and $\epsilon>0$ is arbitrary, this concludes the proof.

In the remaining part of this section we present the simplifications in the proofs of Theorem 1 and Theorem 2 which occur in the free case $q=0$. Here (2) simplifies to

$$
\mathbf{a}_{h} g_{1} \otimes \cdots \otimes g_{n}:=\left\langle h \mid g_{1}\right\rangle g_{2} \otimes \cdots \otimes g_{n}
$$


and the commutation relation (2) reduces to

$$
\mathbf{a}_{g} \mathbf{a}_{h}^{*}=\langle h \mid g\rangle \mathbf{I} .
$$

The scalar product in formula (8) becomes the regular symmetric scalar product in the tensor product of the Hilbert spaces

$$
\left\langle g_{1} \otimes \cdots \otimes g_{n} \mid h_{1} \otimes \cdots \otimes h_{m}\right\rangle= \begin{cases}\prod_{j=1}^{n}\left\langle g_{j} \mid h_{j}\right\rangle & \text { if } m=n \\ 0 & \text { if } m \neq n .\end{cases}
$$

Definition (11) of the Wick product simplifies to

$$
\Psi\left(h \otimes g_{1} \otimes \cdots \otimes g_{n}\right):=\mathbf{X}_{h} \Psi\left(g_{1} \otimes \cdots \otimes g_{n}\right)-\left\langle h \mid g_{1}\right\rangle \Psi\left(g_{2} \otimes \cdots \otimes g_{n}\right) .
$$

From (25) follows the so-called normal ordered representation of Wick products

$$
\Psi\left(g_{1} \otimes \cdots \otimes g_{n}\right)=\sum_{m=0}^{n} \mathbf{a}^{*}\left(g_{1}\right) \ldots \mathbf{a}^{*}\left(g_{n-m}\right) \mathbf{a}\left(g_{n-m+1}\right) \ldots \mathbf{a}\left(g_{n}\right) ;
$$

compare [4, Proposition 1.1]. For example $\Psi(g)=\mathbf{a}_{g}+\mathbf{a}_{g}^{*}, \Psi(f \otimes g)=\mathbf{a}_{f} \mathbf{a}_{g}+$ $\mathbf{a}_{f}^{*} \mathbf{a}_{g}+\mathbf{a}_{f}^{*} \mathbf{a}_{g}^{*}$.

In the proof of Theorem 1 we no longer need to invoke [5, Lemma 1.4] to obtain a bound for the norm of $P^{\otimes n}$, as that is a standard tensor product result [15. Section 2.6.12 Eqn. (16)]. With these simplifications, the proof of Theorem 1 is now self-contained and more transparent.

A key step in the proof of Theorem 2, i.e., (22), can be obtained more directly in the case of free processes. This result can also be derived from Bozejko [3]. We add for completeness the proof in our notation and setting.

Direct proof of (22). Let $\left\{e_{j}: j=1,2, \ldots\right\}$ be an orthonormal basis of $\mathbb{H}$. Then $\left\{e^{\otimes \mathbf{j}}:|\mathbf{j}|=0,1, \ldots\right\}$ forms an orthonormal basis of $\mathcal{H}_{q}$. Since $\mathbf{Z} \in \mathcal{A} \cap \widetilde{\Psi}\left(\overline{\mathbb{H}^{\otimes n}}\right)$, we have the expansion $\mathbf{Z}=\sum_{|\mathbf{i}|=n} \alpha_{\mathbf{i}} \Psi\left(e^{\otimes \mathbf{i}}\right)$. Then

$$
\|\mathbf{Z}\|_{2}=\|\mathbf{Z} \mathbf{1}\|_{\mathcal{H}_{q}}=\left\|\sum_{|\mathbf{i}|=n} \alpha_{\mathbf{i}} e^{\otimes \mathbf{i}}\right\| \|_{\mathcal{H}_{q}}=\left(\sum_{|\mathbf{i}|=n}\left|\alpha_{\mathbf{i}}\right|^{2}\right)^{1 / 2} .
$$

Take $\xi \in \mathcal{H}_{q}$ of norm 1 and expand it into the orthonormal basis

$$
\xi=\sum_{\mathbf{j}} \beta_{\mathbf{j}} e^{\otimes \mathbf{j}}
$$

Using the normal ordered expansion (27) we have

$$
\mathbf{Z} \xi=\sum_{|\mathbf{i}|=n} \sum_{\mathbf{j}} \sum_{m=0}^{n} \alpha_{\mathbf{i}} \beta_{\mathbf{j}} \mathbf{a}_{e_{i(1)}}^{*} \mathbf{a}_{e_{i(n-m)}}^{*} \mathbf{a}_{e_{i(n-m+1)}} \ldots \mathbf{a}_{e_{i(n)}} e^{\otimes \mathbf{j}} .
$$

The expression

$$
\mathbf{a}_{e_{i(1)}}^{*} \mathbf{a}_{e_{i(n-m)}}^{*} \mathbf{a}_{e_{i(n-m+1)}} \ldots \mathbf{a}_{e_{i(n)}} e^{\otimes \mathbf{j}}
$$

is zero, except when the first $m$ components of $\mathbf{j}$ coincide with the last $m$ components of $\mathbf{i}$ in reverse order. Therefore, we keep only the multi-indices in the sum that have the form $\mathbf{i}=\left(\mathbf{i}^{\prime}, \mathbf{k}\right), \mathbf{j}=\left(\overline{\mathbf{k}}, \mathbf{j}^{\prime}\right)$, where $\mathbf{j}^{\prime}$ is arbitrary, $\mathbf{i}^{\prime}$ is an arbitrary multi-index 
of length $\left|\mathbf{i}^{\prime}\right|=n-m, \mathbf{k}$ is arbitrary multi-index of length $|\mathbf{k}|=m$, and $\overline{\mathbf{k}}$ is the reverse of $\mathbf{k}$, i.e., $\bar{k}(s)=k(m-s+1)$. Dropping the primes, we get

$$
\mathbf{Z} \xi=\sum_{m=0}^{n} \sum_{|\mathbf{i}|=n-m} \sum_{\mathbf{j}} \sum_{|\mathbf{k}|=m} \alpha_{(\mathbf{i}, \mathbf{k})} \beta_{(\overline{\mathbf{k}}, \mathbf{j})} e^{\otimes \mathbf{i}} \otimes e^{\otimes \mathbf{j}} .
$$

By the Cauchy-Schwarz inequality for $a_{m} \in \mathbb{C}, m=0,1, \ldots, n$, we have

$$
\left(\sum_{m=0}^{n}\left|a_{m}\right|\right)^{2} \leq(n+1) \sum_{m=0}^{n}\left|a_{m}\right|^{2}
$$

which together with the triangle inequality gives

$$
\|\mathbf{Z} \xi\|_{\mathcal{H}_{q}}^{2} \leq(n+1) \sum_{m=0}^{n}\left\|\sum_{|\mathbf{i}|=n-m} \sum_{\mathbf{j}} \sum_{|\mathbf{k}|=m} \alpha_{(\mathbf{i}, \mathbf{k})} \beta_{(\overline{\mathbf{k}}, \mathbf{j})} e^{\otimes \mathbf{i}} \otimes e^{\otimes \mathbf{j}}\right\|_{\mathcal{H}_{q}}^{2} .
$$

Notice that for a fixed $m \in \mathbb{N}$, different pairs of multi-indices $\mathbf{i}, \mathbf{j}$ of lengths $|\mathbf{i}|=$ $n-m,|\mathbf{j}| \geq 0$ generate different concatenations $(\mathbf{i}, \mathbf{j})$. Thus the corresponding vectors $e^{\otimes \mathbf{i}} \otimes e^{\otimes \mathbf{j}}$ are orthogonal, and we get

$$
\|\mathbf{Z} \xi\|_{\mathcal{H}_{q}}^{2} \leq(n+1) \sum_{m=0}^{n} \sum_{|\mathbf{i}|=n-m} \sum_{\mathbf{j}}\left|\sum_{|\mathbf{k}|=m} \alpha_{(\mathbf{i}, \mathbf{k})} \beta_{(\overline{\mathbf{k}}, \mathbf{j})}\right|^{2} .
$$

By the Cauchy-Schwarz inequality, this gives

$$
\|\mathbf{Z} \xi\|_{\mathcal{H}_{q}}^{2} \leq(n+1) \sum_{m=0}^{n} \sum_{|\mathbf{i}|=n-m,|\mathbf{k}|=m}\left|\alpha_{(\mathbf{i}, \mathbf{k})}\right|^{2} \sum_{\mathbf{j},|\mathbf{k}|=m}\left|\beta_{(\mathbf{k}, \mathbf{j})}\right|^{2} \leq(n+1)^{2}\|\mathbf{Z}\|_{2}^{2}\|\xi\|_{\mathcal{H}_{q}}^{2} .
$$

Therefore (22) follows with constant $C_{q}=1$. The rest of the proof of Theorem 2 then follows unchanged.

\section{Open Questions}

(1) A classical version of a non-commutative process is defined as a classical process that has the same sequence of mixed moments of all orders as the noncommutative process. It would be interesting to clarify if this concept could link Theorem 4 with the Ibragimov conjecture.

(1) Does the $q$-Gaussian sequence in Theorem 4 have a classical version?

(2) If a $q$-Gaussian process is $\psi$-mixing, and has a classical version, does the classical version satisfy the classical $\psi$-mixing condition?

A sufficient condition for the existence of a classical version is given in [5, Section 4]; for a necessary condition, see [10, Theorem 3]. Definitions and properties of the classical (commutative) mixing conditions can be found in $[9]$.

(2) Bradley [8] shows that commutative (not necessarily stationary) Markov chains $X_{k}$ with small values of the $\psi$-mixing coefficient $\psi_{1}$ satisfy a mixing condition which implies that there are positive constants $c, C$ which depend only on $\psi_{1}$ and such that

$$
c \sum E\left(\left|X_{k}\right|^{2}\right) \leq E\left(\left|\sum X_{k}\right|^{2}\right) \leq C \sum E\left(\left|X_{k}\right|^{2}\right) .
$$


Since the Markov property is well-defined in the non-commutative context, it would be interesting to know if Bradley's result, or its implication (28), has a noncommutative version. Theorem 4 shows that without the Markov property the non-commutative version of the left-hand side of (28) fails.

\section{ACKNOWLEDGEMENTS}

The first author thanks M. Bozejko for several discussions, an invitation to a workshop, and references. He also thanks R. Bradley for an early copy of [9], and for encouragement. Both authors benefitted from discussions with M. Peligrad.

\section{REFERENCES}

[1] István Berkes and Walter Philipp. Limit theorems for mixing sequences without rate assumptions. Ann. Probab., 26(2):805-831, 1998. MR 99d:60022

[2] Philippe Biane. Free hypercontractivity. Comm. Math. Phys., 184(2):457-474, 1997. MR 98g:46097

[3] Marek Bożejko. A $q$-deformed probability, Nelson's inequality and central limit theorems. In Garbaczewski and Popowicz, editors, Nonlinear fields: classical, random, semiclassical (Karpacz, 1991), pages 312-335. World Sci. Publishing Co., River Edge, NJ, 1991. MR 93a: 81098

[4] Marek Bożejko. Ultracontractivity and strong Sobolev inequality for $q$-Ornstein-Uhlenbeck semigroup $(-1<q<1)$. Infin. Dimens. Anal. Quantum Probab. Relat. Top., 2(2):203-220, 1999. MR 2002d: 47058

[5] Marek Bożejko, Burkhard Kümmerer, and Roland Speicher. q-Gaussian processes: Noncommutative and classical aspects. Comm. Math. Physics, 185:129-154, 1997. MR 98h:81053

[6] Marek Bożejko and Roland Speicher. An example of a generalized Brownian motion. Comm. Math. Phys., 137(3):519-531, 1991. MR 92m:46096

[7] Richard C. Bradley. A remark on the central limit question for dependent random variables. J. Appl. Probab., 17(1):94-101, 1980. MR 82j:60031

[8] Richard C. Bradley. Every "lower psi-mixing" Markov chain is "interlaced rho-mixing". Stochastic Process. Appl., 72(2):221-239, 1997. MR 98m:60053

[9] Richard C. Bradley. Introduction to strong mixing conditions. Technical report, Indiana University, Bloomington, 2002. ISBN 1-58902-566-0.

[10] Włodzimierz Bryc. Classical versions of $q$-Gaussian processes: conditional moments and Bell's inequality. Comm. Math. Physics, 219:259-270, 2001. MR 2002h:81129

[11] U. Frisch and R. Bourret. Parastochastics. J. Mathematical Physics, 11(2):364-390, 1970. MR 41:4979

[12] I. A. Ibragimov and Ju. V. Linnik. Nezavisimye stalionarno svyazannye velichiny. Izdat. "Nauka", Moscow, 1965. MR 34:2049

[13] I. A. Ibragimov and Yu. V. Linnik. Independent and stationary sequences of random variables. Wolters-Noordhoff Publishing, Groningen, 1971. With a supplementary chapter by I. A. Ibragimov and V. V. Petrov, Translation from the Russian edited by J. F. C. Kingman. MR 48:1287

[14] Marius Iosifescu. Limit theorems for $\phi$-mixing sequences. A survey. In Proceedings of the Fifth Conference on Probability Theory (Braşov, 1974), pages 51-57. Editura Acad. R.S.R., Bucharest, 1977. MR 57:1609

[15] Richard V. Kadison and John R. Ringrose. Fundamentals of the theory of operator algebras. Interscience Publishers, Inc., Academic Press, 1983. MR 85j:46099

[16] A. N. Kolmogorov and Ju. A. Rozanov. On a strong mixing condition for stationary Gaussian processes. Teor. Verojatnost. i Primenen., 5:222-227, 1960. MR 24:A3009

[17] Edward Nelson. Notes on non-commutative integration. J. Functional Analysis, 15:103-116, 1974. MR 50:8102 
[18] Magda Peligrad. An invariance principle for $\phi$-mixing sequences. Ann. Probab., 13(4):13041313, 1985. MR 87b:60056

[19] D. V. Voiculescu, K. J. Dykema, and A. Nica. Free random variables. American Mathematical Society, Providence, RI, 1992. MR 94c:46133

Department of Mathematics, University of Cincinnati, P.O. Box 210025, Cincinnati, Оніо 45221-0025

E-mail address: Wlodzimierz.Bryc@UC.edu

Department of Mathematics, University of Cincinnati, P.O. Box 210025, Cincinnati, Оніо 45221-0025

E-mail address: Victor.Kaftal@UC.edu 